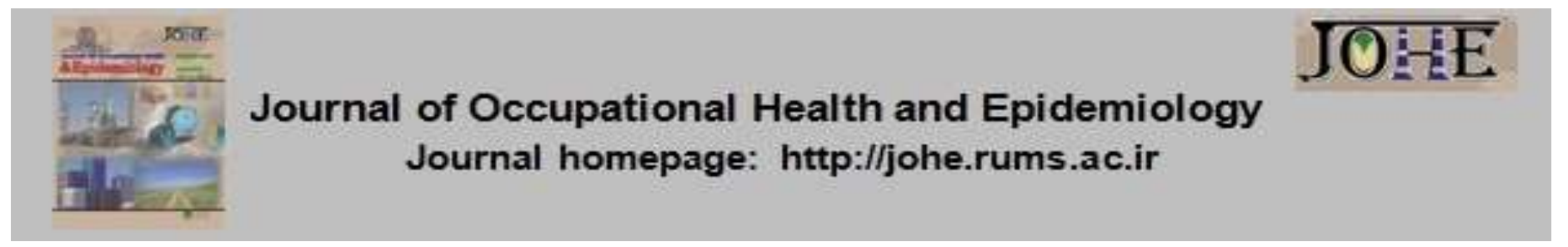

\title{
Is Training Contractors in Safety Issues Effective in Minimizing Occupational Accident Rates? A Retrospective Cohort Study
}

\author{
Zahra Aghajani Aliabadi ${ }^{1}$, Ahmad Soltanzadeh $^{2}$, Samira Ghiyasi ${ }^{3}$
}

1- MSc in Health, Safety, Environment (HSE) Management, Dept. of Environmental Engineering, Central Tehran Branch, Islamic Azad University, Tehran, Iran.

2- Assistant Prof., Dept. of Occupational Safety \& Health Engineering, Research Center for Environmental Pollutants, Faculty of Health, Qom University of Medical Sciences, Qom, Iran.

3- Assistant Prof., Dept. of Environmental Engineering, Central Tehran Branch, Islamic Azad University, Tehran, Iran.

\section{Article Info}

* Corresponding author:

Ahmad Soltanzadeh,

E-mail:

soltanzadeh.ahmad@gmail.com

Article history

Received: Jun 2020

Accepted: Sep 2020

10.29252/johe.9.2.117

Print ISSN: 2251-8096 Online ISSN: 2252-0902

Peer review under responsibility of Journal of Occupational Health and Epidemiology
Citation: Aghajani Aliabadi Z, Soltanzadeh A, Ghiyasi S. Is Training Contractors in Safety Issues Effective in Minimizing Occupational Accident Rates? A Retrospective Cohort Study. JOHE 2020; 9(2):117-23.

\section{Abstract}

Background: Safety of contractors is one of the key safety challenges at construction. Safety training, as an essential prerequisite for achieving safety qualification certificates by contractors, could decrease accident rates in the construction. This study aims to evaluate the effectiveness of training construction contractors in safety issues in minimizing occupational accidents in a five-year period from 2015 to 2019.

Materials and Methods: This is a retrospective cohort study conducted in 2020, recruiting construction contractors. Thirty four contractors had the required safety qualification certificates (qualified), and 28 contractors did not have safety certificates (non-qualified). The analyzed data consisted of demographic information about construction contractors, documents of safety training, contractors' safety qualification certificates, and data on reported occupational accidents. The desirability of safety training was assessed based on a Likert scale questionnaire. IBM SPSS software version 22 was used to analyze the data.

Results: The findings showed that the frequency of occupational accidents was significantly different between qualified and non-qualified contractors $(p<0.05)$. Occupational accidents decreased significantly in the qualified contractors after receiving safety training $(p<0.05)$. The duration of training and the training technique had the highest and lowest desirability, with $80.4 \%$ and $25.3 \%$, respectively.

Conclusions: Although the findings indicated that safety training could have a significant positive effect on minimizing occupational accidents, improving the quantity and quality of safety training parameters, such as the use of up-to-date and applied techniques as well as various media could increase training efficiency.

Keywords: Occupational Accident, Training, Efficiency, Safety, Cohort Study.

\section{Introduction}

The construction industry is one of the main industries that faces daily challenges in terms of safety performance and accidents resulting from safety deficiencies. Research conducted in different countries shows that safety is poorer in the construction industry than in other industries, and many accidents, including minor and major accidents leading to mortality, regularly happen in this industry [1-3].

Contractors are an indispensable part of construction projects and play a major role in the occurrence of occupational accidents in the construction industry. Past research shows that contractors have been involved in occupational accidents occurring in construction projects. 
According to different reports, the highest number of industrial accidents in countries, such as the U.S., China, South Korea, Britain, and Iran, belongs to the construction industry, with many accidents in this field having occurred to seasonal workers and junior contractors [3-7].

Research indicates that some parameters, such as workers' expertise, the level of worker training, and poor safety understanding of construction managers are key factors in the occurrence of accidents in the construction industry. The majority of workers in construction projects are well educated and trained in a specific task, so they are often unfamiliar with other work areas awaiting them in construction projects, which increases the risk of accidents. Insufficient general and subject training could result in the lack of awareness of possible threats and risks, thereby leading to serious adverse consequences in the construction environment. Moreover, lack of sufficient supervision and regular sessions resulting from irresponsibility of managers could cause many types of incidents and increase the risk of accidents [8, 9].

Research results show that safety training and improvement of training indices in the industry could raise awareness among workers and managers of possible risks and dangers in projects. Likewise, some studies indicate that many deficiencies have been observed in the management, training, and attitude of workers, which act as a major accident-creating factor in the industry $[10,11]$.

Although effects of training on reducing occupational accidents have been confirmed by many studies, few studies have reported that such positive effects could not be solely attributed to safety training. Accordingly, there are different parameters, such as the location and time of training, duration of training, quality and quantity of training, as well as the type of training that play a central role in this regard [4, 12]. For instance, Soltanzadeh et al observed that safety training was one of the main factors affecting the occurrence of accidents in the construction industry [13].

Safety qualification certificates are among essential prerequisites for construction companies to participate in tenders; thus, acquisition of such certificates relies on passing safety courses by contracting groups, including contractors, workers, and safety officers. The reason for obtaining such certificates is that workers and contractors need to become acquainted with possible accidents and dangers occurring at construction sites. This, in turn, could improve workers' health and safety and reduce overall costs of contractors. Since worker behavior in project activities is a key factor in many types of occupational accidents, educating workers in occupational dangers and ways of controlling them could considerably improve workers' health and safety. Furthermore, training employers in safety issues could increase their awareness of safety and its consequences, costs of ignoring safety protocols, importance of supplying and using personal protection equipment, and risks associated with construction projects. In addition, it could help contractors understand that training costs are by far lower than those of accidents and subsequent financial losses. Therefore, this study aims to evaluate the effectiveness of training contractors in safety issues in reducing occupational accidents at construction sites.

\section{Materials and Methods}

In the present retrospective cohort study, two groups of qualified and non-qualified construction contractors were investigated. This study was conducted based on data of a five-year period (2015-2019) available for the two groups of contractors, in Tehran province.

Participants of the present study were construction contractors who either had or did not have prerequisite safety qualification certificates. The general directorate of Cooperatives, Labor and Social Welfare in Tehran province provided safety training and granted safety qualification certificates. For the purpose of this study, all contractors who had received safety qualification certificates by 2016 were included in this study as qualified contractors. A total of 36 contractors, including main contractors and subcontractors, were primarily selected for the qualified group. After evaluating their information, 34 contractors having acquired safety qualification certificates were included in the study as final participants in the qualified group. Next, 28 contractors with no safety qualification certificates were randomly selected as non-qualified contractors to be compared with the qualified ones.

Instruments of this study included demographic information of construction contractors, documents of safety training courses, contractors' safety qualification certificates, and data on reported occupational accidents. In addition, to fully acquire the study data on safety training, parameters such as the quantity and quality of the training provided, time and duration of training, as well as training techniques and strategies were employed.

This study was conducted at four stages as follows:

At the first stage, after the inclusion of 62 contractors, data on occupational accidents belonging to the last five years (2015-2019) were 
gathered for the two groups of the study. The data were gathered based on the accident reports extracted from the database of the general directorate of Cooperatives, Labor and Social Welfare in Tehran province as well as the recorded history of accidents reported by the contractors of this study.

At the second stage, the data on the acquisition of safety qualification certificates by construction contractors in the qualified group were collected. Furthermore, the data on the non-qualified contractors who did not have any safety qualification certificates were collected.

At the second stage, data about safety training and its six relevant parameters, including (1) the type of training (involving general safety in construction, personal protective equipment (PPE), industrial housekeeping, work at height, welding, machinery, as well as risk assessment by managers and safety officers), (2) the time of training (morning or afternoon), (3) the duration of training (training workshops running for eight hours, 16 hours, or 20 hours), (4) the quality and content of safety training courses, (5) training techniques (booklets, videos, lectures, discussions, as well as questions and answers), and (6) conditions of the training environment (such as light and temperature) were collected. The desirability of the mentioned parameters was assessed based on a five-point Likert scale (from very low to very good). In addition, the high level of desirability was measured by the two points of good and very good. It should be noted that the desirability of safety training was evaluated based on the results of the questionnaire designed by the general directorate of Cooperatives, Labor, and Social Welfare in Tehran province.

At the fourth stage, the data were analyzed, and the effectiveness of safety training of the contractors in decreasing occupational accidents was evaluated.

To analyze the study data, IBM SPSS software version 22 was used. In addition, the KolmogorovSmirnov test was used to assess the normality of the data. The statistical tests employed were twotailed at the significance level of 0.05 . Besides, the Chi-Square test and the Cochran test were utilized.

\section{Results}

In this study, 34 construction contractors with safety qualification certificates (qualified contractors) and 28 contractors without safety certificates (non-qualified contractors) were evaluated. Among them, 29.4\% of the qualified contractors and $78.6 \%$ of the non-qualified contractors were subcontractors (Table 1).

The results of the comparison between the two groups by the Chi-Square test revealed that there was no significant difference between the qualified and non-qualified contractors in terms of the contract type and the size of contractors ( $p>0.05)$.

Table 1. Demographic information of the two groups

\begin{tabular}{ccccc}
\hline \multicolumn{2}{c}{ Variable } & Qualified contractors & Non-qualified contractors & p-value \\
\hline \multirow{2}{*}{ Contractor type } & Main Contractor & $10(29.4 \%)$ & $6(21.4 \%)$ & \multirow{2}{*}{0.118} \\
\cline { 2 - 4 } & Sub-Contractor ${ }^{\dagger}$ & $24(70.6 \%)$ & $22(78.6 \%)$ & \\
\hline \multirow{2}{*}{$\begin{array}{c}\text { Contractors size } \\
\text { (Number of } \\
\text { workers) }\end{array}$} & $\leq 10$ & $10(29.4 \%)$ & $12(42.9 \%)$ & \multirow{2}{*}{0.072} \\
\cline { 2 - 4 } & $11-25$ & $14(41.2 \%)$ & $4(14.3 \%)$ & \\
\cline { 2 - 4 } & $26-50$ & $8(23.5 \%)$ & $2(7.1 \%)$ & \\
\hline
\end{tabular}

† Contractors with $\leq 25$ workers were considered subcontractors [14].

The results of evaluating safety training desirability in the qualified contractors, based on the analysis of the six safety training parameters evaluated by 1,221 trained people, showed that the highest desirability levels of these parameters were observed for the time of training $(75.9 \%)$, the duration of safety training $(62.7 \%)$, conditions of the training environment (58.6\%), the type of training $(47.0 \%)$, the quality of safety training (46.1\%), and training techniques (31.3\%) (Fig. 1). As Table 2 shows, the analysis of the accident frequency rate in the two groups demonstrated that a total of 723 accidents had happened to the qualified contractors and 889 accidents to the nonqualified ones in the five-year period. The analysis of the occupational accident trend in both groups in five consecutive years by the Cochran test showed that the trend changed significantly in the qualified group ( $p<0.05)$. In contrast, this trend underwent no significant changes in the non-qualified group $(p$ $>0.05$ ). Additionally, the point estimate of the two groups for each of the five years using the ChiSquare test showed that the two groups were not significantly different in 2015 and 2016 ( $p$ > 0.05); however, the changing trend of the occurrence of occupational accidents was significantly different between the two groups in 2017, 2018, and 2019. According to the results, the rate of occupational accidents decreased significantly in the contractors who received safety qualification certificates in the second half of $2016(p<0.05)$. 


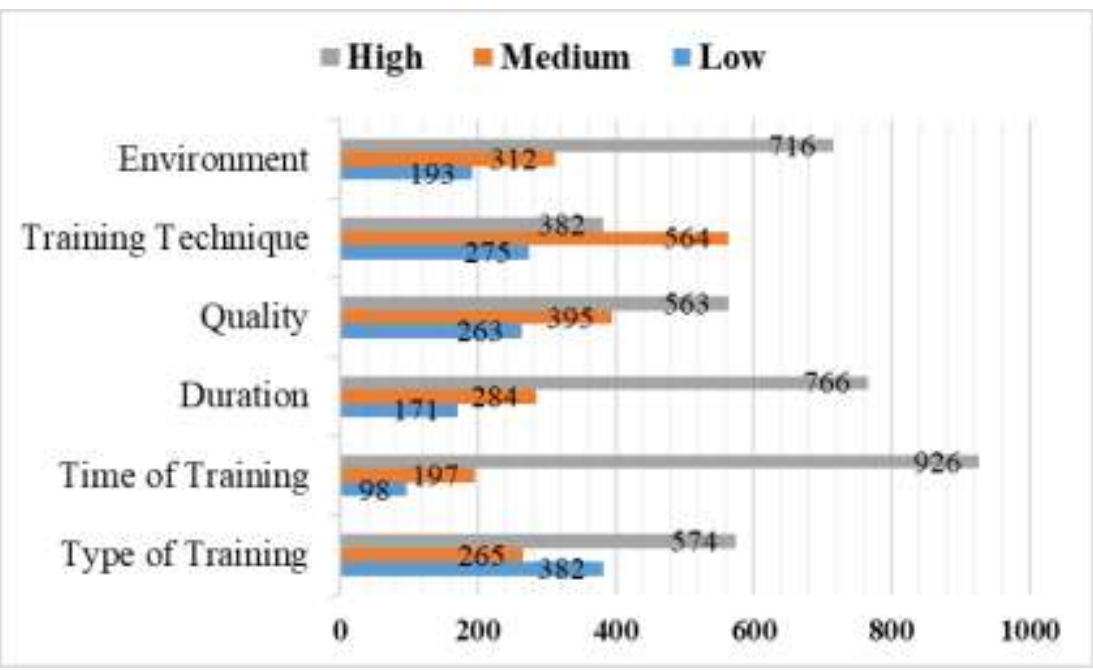

Fig. 1. Assessment results of safety training desirability in the qualified contractors $(n=1221)$

The frequency analysis of mortality in the two groups demonstrated that a total of 30 deaths had occurred among workers of the qualified contractors, and 48 deaths had occurred among workers of the non-qualified contractors in the fiveyear period (Table 2). The analysis of the mortality trend by the Cochran test in both groups in the five consecutive years showed that the trend underwent a significant change in the qualified group $(p<0.05)$. This trend, however, did not change significantly in the non-qualified group $(p>$
0.05). Besides, the point estimate of the mortality trend in the two groups for each of the five years by the Chi-Square test showed that the two groups were not significantly different in 2015 and 2016 (p $>0.05$ ). Nevertheless, there was a statistically significant difference between the two groups in 2017, 2018, and 2019 in the mortality trend $(p<$ 0.05 ). The results also showed that the mortality rate of occupational accidents decreased significantly in the qualified group after the second half of $2016(p<0.05)$.

Table 2. Demographic information of the two groups

\begin{tabular}{ccccc}
\hline \multirow{2}{*}{ Year } & Variable & Qualified ontractors & Non-qualified ontractors & P-value \\
\hline \multirow{2}{*}{2015} & Accident & 199 & 193 & 0.95 \\
\cline { 2 - 5 } & Mortality & 10 & 10 & 1.0 \\
\hline \multirow{2}{*}{2016} & Accident & 187 & 194 & 0.94 \\
\cline { 2 - 5 } & Mortality & 9 & 9 & 1.0 \\
\hline \multirow{2}{*}{2017} & Accident & 138 & 167 & 0.001 \\
\cline { 2 - 5 } & Mortality & 5 & 10 & 0.001 \\
\hline \multirow{2}{*}{2018} & Accident & 108 & 163 & 0.001 \\
\hline \multirow{2}{*}{2019} & Mortality & 3 & 9 & 0.001 \\
& Accident & 91 & 172 & 0.001 \\
\cline { 2 - 5 } & Mortality & 3 & 10 & 0.001 \\
\hline
\end{tabular}

\section{Discussion}

Consistent with the findings of past research on construction safety $[2,12,15,16]$, it was observed in this study that the construction industry and its relevant activities are among the most dangerous parts of industries, and that occupational accidents happening in this environment are mostly of the same nature repeating over and over. One of the main subjects in this regard is the effects of safety issues and problems, such as occupational accidents and their consequences, on construction trends. Research indicates that such effects, studied in terms of different social, humanistic, and economical aspects, are significant [17]. However, the dynamics of construction processes and the provisional nature of construction projects have made it almost impossible for all workers to receive sufficient safety training, thereby leading to poor safety conditions among workers in construction projects. In addition, intense competition among contractors for winning construction tenders has made them pay less attention to some important, yet seemingly costly measures, such as providing workers with appropriate safety training and skills [18].

This study investigated the effects of safety training courses and safety qualification certificates on reducing risks of occupational accidents among construction contractors. To this end, mortality 
data in the field of construction were gathered from 44 contractors in the two groups of qualified contractors with certificates and non-qualified contractors without certificates in a five-year period (2014-2018). According to the findings of this study, the two groups were almost similar in terms of the size and type of the contracting companies with no significant differences in this respect. This parameter played a key role in establishing the validity of the results of this study. The results of the present study showed that the occurrence of occupational accidents and the resulting mortality significantly decreased in the qualified contractors upon the acquisition of safety qualification certificates. This finding stresses the role of safety training in reducing the number of occupational accidents in the construction industry. The results of the point estimate of occupational accidents and the resulting mortality demonstrated that the two groups were not significantly different before the qualified group received safety qualification certificates; however, they became significantly different after the acquisition of the certificates, with the group of the qualified contractors having experienced a significantly lower number of occupational accidents.

Consistent with the findings of this study, past research shows that lack of sufficient awareness of safety issues and protocols among contractors plays a central role in the exhibition of unsafe behaviors and occurrence of occupational accidents. Safety skills could be acquired by the combination of safety parameters. Workers facing various types of occupational dangers at construction sites should be well educated and be able to use safety awareness at work. Furthermore, training courses need to be updated so as to raise awareness of safety protocols and occupational dangers among contractors and workers. Lack of training and insufficient safety training among workers, which include a wide range of training parameters, such as the type of training, time and duration of training, content of training courses, training techniques, and training conditions, are among the main reasons for occupational accidents. A worker not having passed any safety training courses either through safety training classes or written safety instructions will be unable to realize occupational dangers and to avoid negative consequences [19, 20].

Several studies have reported that insufficient, inappropriate, and inefficient training could result in carelessness, dangerous behavior, and different types of human errors in construction projects. In the same vein, some studies have reported that identification of dangers and risk awareness could be improved by training interventions. Thus, paying full attention to safety training and improving training indices in the construction industry could lead to a better understanding of occupational dangers and risks, thereby enhancing safety in construction projects [21, 22].

The results of this study, in general, indicate that it is essential to focus on safety training, qualification, and awareness among construction contractors, senior and junior workers, as well as safety officers. Although general safety training exerts insignificant effects on reducing occupational dangers and risks in the construction industry, specific skill-based safety training, especially industrial housekeeping, could make construction stakeholders aware of dangers and risks in construction projects [23, 24]. Research reports that safety training programs could exert a positive impact on safety behaviors among contractors and workers in some construction projects $[11,25,26]$. Another key factor in preventing occupational accidents and work injuries is to ensure that safety culture is appreciated in construction projects. Lack of management accountability, required connections, an atmosphere of participation and support among workers, as well as workers' attitudes and qualifications, being a consequence of inefficient safety training, has been considered by some studies to act as an obstacle to fostering safety culture in construction projects $[27,28]$. Therefore, based on the findings of this study, safety training could result in an accepted level of desirability, thereby reducing occupational accidents and mortality rates in construction projects.

Accordingly, due to the limitations of this study, such as the small sample size, lack of access to data, such as training effectiveness parameters, and limitations in the geographical area of the study, the authors suggest that future research be conducted with a larger sample size. In addition, it is suggested that relevant parameters and variables be modelled to evaluate the efficiency, effectiveness, and productivity of safety training.

\section{Conclusion}

Although the findings of this study indicate that safety training could minimize the occurrence of occupational accidents, paying attention to important parameters and indicators of training, such as the quantity and quality of safety training at construction sites, could improve the efficiency of training and increase productivity in construction projects. Therefore, it is suggested that safety training be identified, designed, and provided based on the assessment of requirements and benchmarking. Thus, it is suggested that a reactive 
approach be adopted, which involves the reviewing of past accidents and losses in construction projects, as well as other proactive approaches, such as risk assessment and hazard identification in the construction industry. In addition, it should be noted that designing an algorithm for safety training in the construction industry should fit characteristics and limitations of construction projects as well as features of human resources involved in these projects.

\section{Acknowledgement}

The authors of the present research would like to extend their sincere gratitude to all contractors who participated in this study for their invaluable and professional assistance.

Conflict of interest: None declared.

\section{References}

1. Mohammadfam I, Soltanzadeh A, Moghimbeigi A, Akbarzadeh $M$. Factors affecting occupational accidents in the construction industry (2009-2013). Journal of Occupational Health and Epidemiology 2014; 3(2):88-95.

2. Soltanzadeh A, Mohammadfam I, Moghimbeygi A, Ghiasvand R. Exploring Causal Factors on the Severity Rate of Occupational Accidents in Construction Worksites. International Journal of Civil Engineering 2017; 15(7):959-65.

3. Zhang F, Fleyeh H, Wang X, Lu M. Construction site accident analysis using text mining and natural language processing techniques. Automation in Construction 2019; 99:238-48.

4. Soltanzadeh A, Mohammadfam I, Moghimbeigi A, Ghiasvand R. Key factors contributing to accident severity rate in construction industry in Iran: a regression modelling approach. Arch Hig Rada Toksikol 2016; 67(1):47-53.

5. Alizadeh Savareh B, Mahdinia M, Ghiyasi S, Rahimi J, Soltanzadeh A. Accident Modeling in Small-scale Construction Projects Based on Artificial Neural Networks. Journal of Human Environment and Health Promotion 2019; 5(3):121-6.

6. Kang K, Ryu H. Predicting types of occupational accidents at construction sites in Korea using random forest model. Saf Sci 2019; 120:226-36.

7. Passmore D, Chae C, Borkovskaya V, Baker R, Yim JH. Severity of U.S. Construction Worker Injuries, 2015-2017. E3S Web Conferences 2019; 97:06038. doi:10.1051/e3sconf/20199706038

8. Prasad R. Selection of internal safety auditors in an Indian construction organization based on the SWARA and ARAS methods. Journal of Occupational Health and Epidemiology 2019; 8(3):134-40.

9. Soltanzadeh A, Mohammadfam I, Moghimbeigi A. P153 Predicting and determining factors of occupational accidents severity rate (ASR) using artificial neural networks (ANN); a case study in construction industry. Occup Environ Med 2016; 73(Suppl 1):A171-2.

10. Soltanzadeh A, Derakhshan Jazari M, Heidari $\mathrm{H}$, Mohammadi H, Mohammadbeygi A. Modeling Causal Factors of Occupational Accidents in Chemical Industries: A 10-Year Field Study in Iran. Iranian Journal of Chemistry and Chemical Engineering; 2019 Oct 8. doi: 10.30492/ijcce.2019.36824

11. Zamanian Z, Mehrifar Y. Study Health and Safety Workplace in Small and Medium Size Enterprises (HSW-SMEs) in Iran, 2015. Journal of Occupational Health and Epidemiology 2016; 5(2):112-20.

12. Ayhan BU, Tokdemir OB. Accident Analysis for Construction Safety Using Latent Class Clustering and Artificial Neural Networks. J Constr Eng Manag 2020; 146(3). doi:10.1061/(ASCE)CO.1943-7862.0001762

13. Soltanzadeh A, Heidari $H R$, Mahdinia $M$, Mohammadi $\mathrm{H}$, Mohammadbeygi $\mathrm{A}$, Mohammadfam I. Path analysis of occupational injuries based on the structural equation modeling approach: a retrospective study in the construction industry. Iran Occupational Health 2019; 16(3):47-57.

14. Manu E, Ankrah N, Chinyio E, Proverbs D. Trust influencing factors in main contractor and subcontractor relationships during projects. International Journal of Project Management 2015; 33(7):1495-508.

15. Choi SD, Guo L, Kim J, Xiong S. Comparison of fatal occupational injuries in construction industry in the United States, South Korea, and China. International Journal of Industrial Ergonomics 2019; 71:64-74.

16. Zamanian Z, Hashemi H, Azad P, Mehri Y, Kohnavard B. Survey on factors affecting occupational accidents among construction industry workers in Khorramabad, Iran. Journal of Occupational Health and Epidemiology 2014; 3(1):26-31.

17. Ling FY, Zhang Z, Guo L. Measures to regain productivity after construction accidents. In: Creative Construction Conference 2019. Proceedings of the Creative Construction Conference 2019; 2019 June 29 - July 2; Budapest, Hungary. Budapest: University of Technology and Economics of Budapest; 2019. P. 719-24.

18. Borkovskaya V, Lyapuntsova E, Nogovitsyn M. Risks and safety in construction by increasing efficiency of investments. E3S Web of Conferences 2019; 97:06036. doi: 10.1051/e3sconf/20199706036

19. Min K, Cha Y, Han S, Hyun C. An Analysis of Relationship between Unsafe Acts and Human Errors of Workers for Construction Accident Prevention. Journal of the Architectural Institute of Korea Structure \& Construction 2019; 35(5):161-8. 
20. Ye G, Tan Q, Gong X, Xiang Q, Wang Y, Liu Q. Improved HFACS on Human Factors of Construction Accidents: A China Perspective. Advances in Civil Engineering 2018; 2018:4398345. doi: 10.1155/2018/4398345

21. Boamah FA. Strategies for curbing ineffective management of safety on construction sites: A case study in the Greater Accra Region [MSc thesis]. Kumasi, Ghana: Kwame Nkrumah University of Science and Technology; 2016.

22. Fazli B, Ansari H, Zare H, Hami Mahkoyeh S, Sadeghzadeh A, Fazli F, et al. Safety culture in GolGohar Mine in Southeast Iran in 2014. Journal of Occupational Health and Epidemiology 2014; 3(2):117-25.

23. Alruqi WM, Hallowell MR. Critical Success Factors for Construction Safety: Review and Meta-Analysis of Safety Leading Indicators. J Constr Eng Manag 2019; 145(3). doi: 10.1061/(ASCE)CO.1943-7862.0001626

24. Kiani F, Khodabakhsh MR. The role of supervisor in effectiveness of safety training session and changing employees' attitudes toward safety issues. Journal of Safety
Promotion and Injury Prevention 2015; 3(1):4956.

25. Dale AM, Buckner-Petty S, Evanoff B. P. 1.08 Connecting contractor safety management programs and worker perceived safety climate in commercial construction projects. Occup Environ Med 2019; 76(Suppl 1):A79-80.

26. Okonkwo PN, Wium J. Health and Safety Management Systems within Construction Contractor Organizations: Case Study of South Africa. J Constr Eng Manag 2020; 146(5). doi: 10.1061/(ASCE)CO.1943-7862.0001833

27. Trinh MT, Feng Y. Impact of Project Complexity on Construction Safety Performance: Moderating Role of Resilient Safety Culture. J Constr Eng Manag 2020; 146(2). doi: 10.1061/(ASCE)CO.1943-7862.0001758

28. Al-Bayati AJ, Albert A, Ford G. Construction Safety Culture and Climate: Satisfying Necessity for an Industry Framework. Practice Periodical on Structural Design and Construction 2019; 24(4). doi: 10.1061/(ASCE)SC.1943-5576.0000452 